\title{
Smoking Cessation Intervention: The Practices of Maternity Care Providers
}

\author{
Amy L. Helwig, MD, Geoffrey R. Swain, MD, and Mark Gottlieb, PbD
}

Background: Little is known about the actual smoking intervention practices of maternity care providers. This study examines smoking intervention practices of maternity care providers in a large Midwestern city.

Methods: A telephone survey was used to collect information on the care of pregnant patients who smoke $(\mathbf{n}=73)$.

Results: After brief counseling during the first prenatal care visit ( 98 percent), 84 percent of providers readdressed smoking at follow-up visits. Family physicians were significantly more likely than obstetricians to provide further counseling ( 97 versus 72 percent, $P<0.005$ ). Common cessation interventions included provider counseling ( 99 percent) and recommending smoking cessation classes ( 26 percent). Reasons cited by providers for patients' inability to stop smoking during pregnancy included addiction, habit, and environment or family. Methods used to encourage smoking cessation did not correlate with those known to be effective for treating addiction, modifying behavior, or responding to family or social system problems.

Conclusion: Maternity care providers underutilize effective methods of smoking cessation for their patients who smoke and rely on less effective methods. (J Am Board Fam Pract 1998;11:336-40.)

Twenty to 40 percent of US women of reproductive age currently smoke, and among pregnant women the overall prevalence of smoking is 19.1 percent. ${ }^{1,2}$ Because smoking significantly increases maternal and fetal morbidity and mortality, reducing the number of pregnant smokers has been a high public health priority since the 1970 s. $^{3}$

A number of studies have tested the effectiveness of different smoking cessation methods in pregnant women, comparing these methods with the usual care of physician advice alone or referral to smoking cessation classes. In one study none of the 112 women referred to smoking cessation classes actually attended the class. ${ }^{4}$

The quit rates for usual care for pregnant women range from 0 to 3 percent. ${ }^{5-8}$ This rate compares poorly to quit rates of alternative programs such as educational videotapes (19 percent $),{ }^{5}$ individual counseling by trained counselors (9 to 15 percent), ${ }^{6,9}$ pregnancy-specific self-help booklets or manuals (14 to 17 percent), 8,10 and multiple component programs (11 to 32 percent). ${ }^{11-14}$ The latter combined mailings, personal

Submitted, revised, 12 January 1998.

From the Columbia Family Practice Residency, Medical College of Wisconsin (ALH, GRS, MG) and the City of Milwaukee Health Department (GRS), Milwaukee. Address reprint requests to Amy Helwig, MD, Columbia Family Practice Residency, Medical College of Wisconsin, 210 West Capital Dr, Milwaukee, WI 53212. visits, and additional telephone contacts. Even nicotine replacement therapy (a pregnancy category $\mathrm{D}$ substance that should be used only if the benefits outweigh the risks) has been studied, albeit in women who failed customary smoking cessation efforts. ${ }^{15}$ Further, special interventions among those who have quit can decrease the rate of relapse as well. ${ }^{16}$

Two special smoking cessation interventions reported quit rates above 50 percent: the Indiana State Department of Health Prenatal Use Prevention Program, ${ }^{17}$ and a University of Vermont program that provided special training for obstetric and family practice residents in giving brief, structured advice to their smoking prenatal patients. ${ }^{18}$ Additionally, smoking cessation efforts in pregnant women have been shown to be cost-effective. ${ }^{12}$

Despite our knowledge of the occurrence of smoking during pregnancy, its related health consequences, and the success rates and cost-effectiveness of smoking cessation interventions, very little is known about the actual smoking intervention practices of maternity care providers. Is the poorly effective usual care actual practice? Three independent literature searches revealed almost no information on this subject. An Australian study of public antenatal clinic medical and nursing directors showed that only 12 percent of clinics offered training to their staff on smoking cessation and only 4 percent had a written policy on the issue. ${ }^{19}$ 
The objective of our study was to investigate the usual intervention practices of family physicians, obstetricians, and nurse midwives for their patients who smoke.

\section{Methods}

We developed a telephone survey to assess the smoking cessation management techniques used by maternity care providers for pregnant patients. The survey was designed to answer the following questions: (1) To what extent do health care providers address smoking status with their pregnant patients? (2) What types of interventions do providers use to encourage pregnant women to stop smoking? (3) What do providers perceive as the reason their pregnant patients continue to smoke? (4) Is there a difference in smoking cessation interventions based on specialty of the health care provider? We also gathered background information, including provider specialty, age, number of pregnant patients per year, type of practice (group, solo, health maintenance organization [HMO], academic), and third-party payer status. Providers were asked whether they discussed smoking with their pregnant patients, what types of interventions they used to encourage pregnant women to stop smoking, whether they readdressed smoking at later prenatal care visits with women who are known to smoke, and what they perceived as the greatest barrier their pregnant patients faced when trying to quit smoking. We asked providers to discuss only their management of smoking in pregnant women, not in their general patient population.

The survey population included all maternity care providers in a large Midwestern city (population 638,000). Providers were selected from obstetricians, family physicians, and midwives listed in the telephone book and hospital directories, assuring that patients from all parts of the city and all socioeconomic groups were included. The offices of all family physicians in the city were contacted to determine whether the physicians actively provided obstetric services. Family physician participants were limited to those who maintained an obstetric practice.

The survey was conducted during regular office hours by a third-year family practice resident. Response rates were analyzed using chisquare and Fisher's exact tests with the STATA software package. ${ }^{20}$

\section{Results}

Seventy-five obstetricians (45 offices), 44 family physicians actively practicing obstetrics (22 offices), and 14 certified nurse midwives ( 5 offices) were selected as the potential survey contacts. From this population 73 providers were contacted by telephone during their office hours. One of the contacts, an obstetrician, declined to participate. Thirty-three obstetricians, 33 family physicians, and 6 certified nurse midwives completed the survey for a total of 72 participants. Because of time limitations, only 1 provider per office was interviewed. A provider was contacted in 91 percent of the family physician practices offering maternity care, 67 percent of the obstetric practices, and 100 percent of the certified nurse midwife practices. Of the providers surveyed, 62 percent were in group practice, 33 percent in solo practice, and 3 percent in a staff model HMO. Contacts were made in three family practice residencies and two obstetrics-gynecology residencies, with contacts including 1 senior resident and 1 faculty member for each program.

At the initial visit all but 1 provider, an obstetrician, discussed smoking with pregnant patients (98 percent), and 84 percent of all providers followed through with discussion at subsequent prenatal visits. Thirty-two family physicians (97 percent) reported follow-up attention to smoking, significantly more than the 24 obstetricians ( 72 percent; $P<0.005)$. All midwives ( 100 percent) readdressed smoking at later prenatal visits. Twenty-four percent of family physicians and 15 percent of obstetricians reported that they bring up smoking at all prenatal visits for their patients who smoke.

After general counseling ( 99 percent of all providers), the next most frequently reported intervention was referral to smoking cessation classes by 33 percent of family physicians, 18 percent of obstetricians, and 33 percent of midwives; differences were not statistically significant. Among all providers, other reported interventions included nurse counseling (19 percent), health educator counseling ( 7 percent), relaxation and stress management techniques ( 10 percent), brochures and educational materials ( 14 percent), and nicotine skin patch or gum substitutes (11 percent). No significant differences were found among provider groups in the use of these methods; however, obstetricians tended to offer more interventions than family physicians. The midwives used only two ad- 
ditional interventions other than provider counseling: smoking cessation classes and health educators. Most ( 89 percent) maternity care providers surveyed correctly considered nicotine substitutes to be contraindicated during pregnancy, although these providers prescribed the substitutes for nonpregnant patients.

Providers were also asked to describe the major reason why their patients were not able to stop smoking during pregnancy (open-ended format). Among all providers, the primary perceived reasons for continued patient smoking during pregnancy included addiction (49 percent), habit (19 percent), and environment or family (lack of family support and other smokers in the home) (11 percent). Family physicians ( 84 percent) tended to mention habit and addiction as barriers more frequently than did obstetricians (62 percent) or midwives ( 17 percent). Of the less frequently cited barriers, family issues were cited more often by midwives ( 33 percent) and family physicians (15 percent), whereas obstetricians more often cited lack of motivation (15 percent), apathy (12 percent), and peers (12 percent). Other occasionally cited barriers included stress, education, and lifestyle. No statistically significant differences were found among the different maternity care providers. In addition, no association was found between the providers' perceptions of barriers to smoking cessation and the type of interventions they offered patients.

\section{Discussion}

Pregnancy offers a strong incentive to quit smoking, evidenced by one study showing that 41 percent of women stopped smoking with an initial diagnosis of pregnancy. ${ }^{13}$ In addition, pregnant women in smoking cessation programs move more rapidly through stages of change (eg, precontemplation, contemplation) than do pregnant women who receive only usual care, especially during the first trimester. ${ }^{21}$ More than one quarter of obstetricians in our survey, however, made only a single attempt to discuss smoking cessation with pregnant patients who smoked and did not follow through with smoking cessation support during subsequent prenatal visits. Reasons for the significant difference in attempted follow-up intervention between obstetricians ( 72 percent) and family physicians ( 97 percent) are not known. All midwives pursued smoking cessation with their clients; again, the reason why is unknown. Possible explanations for these findings include variations in practice orientation toward continuity of care and prevention or simply providers' differing personal perceptions of the value of such followup. Interspecialty differences have been noted previously in other aspects of obstetric care, so our findings are not surprising. ${ }^{14}$

Our findings also suggest that, as a group, maternity care providers underutilize effective smoking cessation methods in pregnant women, relying instead on methods that are only minimally effective. While recommending stopping smoking and sometimes referring patients to a smoking cessation group reflect the usual care of pregnant women who smoke, they are not the most effective interventions. Numerous studies show that interventions beyond physician counseling and class referral have a positive impact on pregnant women's smoking cessation rates. ${ }^{5-12,15-19,22,23}$ Interventions such as personal visits, mailings, videotapes, selfhelp manuals, and extensive counseling by trained health educators or nurses were found to be both cost-effective and feasible. Nevertheless, general counseling, or provider discussion, was the preferred method of intervention in this study. Approximately one third of all providers in our study referred patients to smoking cessation classes or educators, less than 20 percent used educational materials, and only about 10 percent used relaxation and stress management techniques or nicotine replacement substitutes.

In addition to underutilizing smoking cessation methods that have been shown to be more effective, the providers in our study used methods that were not in concordance with their perceptions of the reasons patients cannot stop smoking. This contradiction suggests several possibilities. Providers themselves might lack appropriate education about smoking cessation interventions and their efficacy. Appropriate programs or reimbursement mechanisms might not be available. Providers might not have experienced success with offering smoking cessation to patients and might be cynical about the prospects of behavior change. Finally, providers might not be aware of the strength of pregnancy as a motivator, albeit perhaps temporary, for smoking cessation.

This study has several limitations. Findings in one city might not be generalizable to another city. The number of survey contacts was limited by the 
time available to complete the survey. Physician's self-reported behavior might not correlate well with the objectively measured practice. Social bias could be present in this survey, which was a physician-to-physician interview. This bias would likely have increased the providers' self-reports of intervention. In addition, some bias could have resulted from the use of a telephone survey. Those providers who were available in their office during the hours the telephone interview was performed might have caused some selection bias. There were few midwives surveyed, as their presence in maternity care is not large in this community.

\section{Conclusions}

Our survey results indicate that maternity care providers might not be taking advantage of the motivation pregnancy affords or the most effective smoking cessation methods to help pregnant women stop smoking. Sixteen percent of providers did not continue to address smoking during prenatal visits, and smoking cessation interventions known to be effective were used with frequencies of less than 20 percent.

There are many effective approaches available to prenatal care providers. These approaches include mailings, videotapes, self-help materials, and counseling by trained nurses and health educators. To begin to reach year 2000 goals, providers should include at least one of these approaches in addition to their usual care. Ideally, a multidisciplinary approach would be available to all pregnant women who smoke. Increased utilization of effective smoking cessation programs, more physician education, better reimbursement for existing smoking cessation programs, and additional funded programs for pregnant women who smoke are also needed. Further study is merited to determine which factors limit providers' ability to offer more extensive or appropriate smoking cessation options to pregnant women.

Family physicians have a special opportunity not only to encourage and support pregnant women to stop smoking during pregnancy but also to continue to enforce this message during the postpartum period and at well-child visits, a time when many women who quit smoking easily relapse. Obstetricians might not have as many long-term opportunities to help patients stop smoking, but they too could improve their interventions during the course of a pregnancy.

\section{References}

1. Anonymous. Pregnancy risks determined from birth certificate data - United States, 1989. MMWR Morbid Mortal Wkly Rep 1992;41:556-63.

2. Windsor RA, Orleans CT. Guidelines and methodological standards for smoking cessation intervention research among pregnant women: improving the science and art. Health Educ Q 1986:13:131-61.

3. Windsor RA, Lowe JB, Perkins LL, Smith-Yoder D, Artz L, Crawford M, et al. Health education for pregnant smokers: its behavioral impact and cost benefit. Am J Public Health 1993;83:201-6.

4. O'Connor AM, Davies BL, Dulberg CS, Buhler PL, Nadon C, McBride BH, et al. Effectiveness of a pregnancy smoking cessation program. J Obstet Gynecol Neonatal Nurs 1992;21:385-92.

5. Secker-Walker RH, Solomon LJ, Geller BM, Flynn BS, Worden JK, Skelly JM, et al. Modeling smoking cessation: exploring the use of a videotape to help pregnant women quit smoking. Women Health. 1997;25:23-35.

6. Secker-Walker RH, Solomon LJ, Flynn BS, Skelly JM, Lepage SS, Goodwin GD, et al. Individualized smoking cessation counseling during prenatal and early postnatal care. Am J Obstet Gynecol 1994;171: 1347-55.

7. Mayer JP, Hawkins B, Todd R. A randomized evaluation of smoking cessation interventions for pregnant women at a WIC clinic. Am J Public Health 1990;80:76-8.

8. Windsor RA, Warner KE, Cutter GR. A cost-effective analysis of self-help smoking cessation methods for pregnant women. Public Health Rep 1988;103: 83-8.

9. Dunkley J. Training midwives to help pregnant women stop smoking. Nurs Times 1997;93:64-6.

10. Windsor RA, Cutter G, Morris J, Reese Y, Manzella $\mathrm{B}$, Bartlett EE, et al. The effectiveness of smoking cessation methods for smokers in public health maternity clinics: a randomized control trial. Am J Public Health 1985;75:1389-92.

11. Duffy J, Coates TJ. Reducing smoking among pregnant adolescents. Adolescence 1989;24:29-37.

12. Dolan-Mullen P, Ramirez G, Groff JY. A metaanalysis of randomized trials of prenatal smoking cessation interventions. Am J Obstet Gynecol 1994; 171:1328-34.

13. O'Campo P, Faden RR, Brown H, Gielen AC. The impact of pregnancy on women's prenatal and postpartum smoking behavior. Am J Prev Med 1992;8:813.

14. Rosenblatt RA, Dobie SA, Hart LG, Schneeweiss R, Gould D, Raine TR, et al. Interspecialty differences in obstetric care of low-risk women. Am J Public Health 1997;87:344-51.

15. Wright LN, Thorp JM Jr, Kuller JA, Shrewsbury $\mathrm{RP}$, Anath C, Hartmann K. Transdermal nicotine replacement in pregnancy: maternal pharmacokinetics and fetal effects. Am J Obstet Gynecol 1997;175: $1090-4$. 
16. Secker-Walker RH, Solomon LJ, Flynn BS, Skelly JM, Lepage SS, Goodwin GD, et al. Smoking relapse prevention during prenatal and early postnatal care. Am J Prev Med 1995;11:86-93.

17. Waller CS, Zollinger TW, Saywell RW Jr, Kubisty $\mathrm{KD}$. The Indiana prenatal substance use and prevention program: its impact on smoking cessation among high-risk women. Indiana Med 1996;89:184-7.

18. Secker-Walker RH. Solomon LJ. Flynn BS. LePage SS, et al. Training obstetric and family practice residents to give smoking cessation advice during prenatal care. Am J Obstet Gynecol 1992;166:1356-63.

19. Walsh RA, Redman S, Birnsmead MW, Arnold B. Smoking cessation in pregnancy: a survey of the medical and nursing directors of public antenatal clinics in Australia. Aust N Z J Obstet Gynaecol 1995;35:144-50.

20. STATA statistical software, release 5.0. College Station, Tex: STATA Corp, 1997.

21. Solomon LJ, Secker-Walker RH, Skelly JM, Flynn BS. Stages of change in smoking during pregnancy in low-income women. J Behav Med 1996;19:33344.

22. Tyley T. Cigarette smoking in pregnancy. Modern Midwife 1996;6:17-9.

23. Kilby JW. A smoking cessation plan for pregnant women. J Obstet Gynecol Neonatal Nurs 1997;26: $397-402$. 